\title{
Synthesis and herbicidal activity of 1,3-substituted quinazolinedione derivatives
}

\author{
Hai-Bo Yu, Hong-Fei Wu, Dong-Liang Cui, and Bin Li* \\ Shenyang Research Institute of Chemical Industry, Shenyang, 110021, PRC \\ E-mail: libinjia@yahoo.com.cn
}

\begin{abstract}
Quinazolinediones derivatives are a new family of diphenyl ether herbicides. Twenty four 1,3substituted quinazolinediones derivatives were designed and synthesized. Their structures were confirmed by ${ }^{1} \mathrm{H}$ NMR and elemental analysis. The preliminary bioassay results show that most of these compounds have herbicidal activity in post-emergence treatment. The compounds $3 \mathrm{c}$, 3d, 3e, 3f showed 90-100\% herbicidal efficacy at 200 gha $^{-1}$ against dicotyledonous weeds such as Abutilon theophrasti.
\end{abstract}

Keywords: Quinazolinediones, herbicidal activity, diphenyl ether

\section{Introduction}

Many herbicides have recently been phased out because of their potential toxicological or environmental impact. These side effects are often caused by high use-rates. Thus, products such as protoporphyinogen-IX oxidase (protox) inhibitors, which can control weeds at a low use-rate of a few gha ${ }^{-1}$, are of top interest. Protox is the last enzyme in the porphin pathway that is common to both chlorophyll and heme synthesis. In treated tissues, these herbicides cause membrane leakage, pigment breakdown and eventually necrosis of the leaf. ${ }^{1-3}$

Research on protox inhibitors has been actively pursued since the 1970s, producing roughly two classes of product: diphenyl ethers (DPEs) and $N$-phenylnitrogen heterocycles. ${ }^{4}$ DPEs were the first widely used family of herbicides. Several novel heterocyclic phenyl ethers have been reported by several groups (Fig. 1 and Fig. 2).$^{5-8}$ Heterocyclic phenyl ethers are highly active both pre- and postemergence on a wide variety of weeds. ${ }^{9-17}$ A few recently reported examples include 6-aryloxy-1 $H$-benzotriazoles, ${ }^{18}$ aryloxyindolin-2-( $3 H$-ones, 5 -aryloxybenzisoxazoles, 6aryloxyquinoxalin-2,3-diones, benzoheterocycles, ${ }^{19-21}$ and benzoxazines. ${ }^{22}$

Through studying the structure-activity relationships of heterocyclic phenyl ethers, we added an ester or an acylamide on N-3 of quinzaolinediones to improve the activity. In this paper, we 
describe synthesis of 1,3-substituted quinazolinediones derivatives and their structure-activity relationships for herbicidal activity.
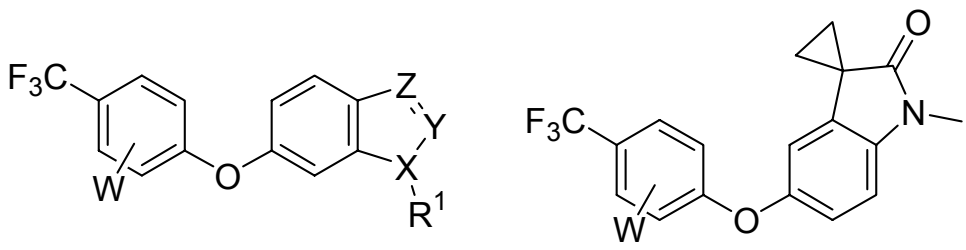

$\mathrm{X}, \mathrm{Y}, \mathrm{Z}=\mathrm{C}, \mathrm{N}, \mathrm{O} \quad \mathrm{W}=\mathrm{H}, \mathrm{Cl}, \mathrm{F}$

Figure 1. Structural characteristics of benzoheterocyclic analogs.<smiles>[X]c1cc(C(F)(F)F)cc(Cl)c1Oc1ccc2c(c1)OC([R16])([R])C(=O)N2[R7]</smiles><smiles>[X]c1cc(C(F)(F)F)cc(Cl)c1Oc1ccc2c(c1)N(C)C(=O)C(C)O2</smiles><smiles>Cn1c(=O)c2cc(Oc3ccc(C(F)(F)F)cc3Cl)ccc2n(C)c1=O</smiles>

Figure 2. Structural characteristics of benzoxazines and quinazolinediones.

\section{Results and Discussion}

2-(2-Amino-5-(2-chloro-4-4(trifluoromethyl)phenoxy)benzamido) acetate (1, $\mathrm{R}^{1}$ was $\mathrm{H}$ or methyl, $\mathrm{R}^{2}$ was methyl or ethyl), obtained via multistep synthesis, ${ }^{23,24}$ were treated triphosgene with at $0{ }^{\circ} \mathrm{C}$ and $\mathrm{Et}_{3} \mathrm{~N}$ as the base, to produce 2-(6-(2-chloro-4-4(trifluoromethyl)phenoxy)-2,4dioxo-1,2-dihydroquinzaolin-3(4H)-yl) acetate $(2)^{25}$. The hydrogen atom was substituted by methyl, allyl or propargyl to give (3). The ester was hydrolyzed to afforded (4), which was converted to various amide groups using standard amidation methods to provide the 2-(1substituted-2,4-dioxoquinazolin-3(4H)-yl)- $N$-substituted acetamides (5) (Scheme 1).

Through the herbicidal activity testing, we found that the benzoquinazoline derivatives were more active on dicotyledonous than monocotyledonous species (Table 1). Some compounds showed excellent herbicidal activity at the rate of $200 \mathrm{gha}^{-1}$ such as, compound 3c, 3d, 3e, 3f, Which have a better herbicidal activity compare to the related compound 1,3-substituted quinazolinedione reported in the literature (the reported compounds show low herbicidal activity at the same rate). The order of herbicidal activity of benzoquinazoline compounds was as follows: $(3)>(2)>(4)>(5)$. The activity of the ester was greater than acid and acylamide. Some of the compounds exist in enantiomeric forms, their herbicidal activity were similar. While $\mathrm{R}^{3}$ was substituted with propynyl, allyl, the compounds showed good herbicidal activity. Activities may 
be characterized with propynyl or allyl group because the higher hydrophobicity, which affecting the uptake, translocation or metabolism in the plant.<smiles>[R]OC(=O)C([R1])NC(=O)c1cc(Oc2ccc(C(F)(F)F)cc2Cl)ccc1N</smiles>

1

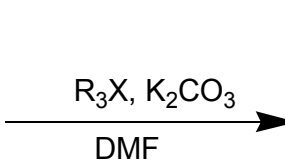<smiles>[R2]OC(=O)C([R7])n1c(=O)[nH]c2ccc(Oc3ccc(C(F)(F)F)cc3Cl)cc2c1=O</smiles>

2

3<smiles>[R3]C(C(=O)O)n1c(=O)c2cc(Oc3ccc(C(F)(F)F)cc3Cl)ccc2n([R3])c1=O</smiles>

4

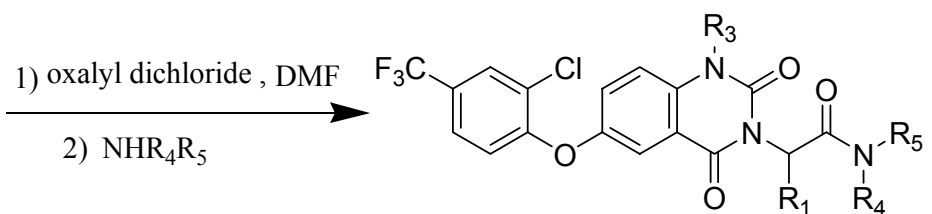

5

Scheme 1. Synthesis route for 1,3-substituted quinazolinediones derivatives.

\section{Experimental Section}

General Procedures. Melting points were measured by using a RY-1 melting point apparatus and are uncorrected. ${ }^{1} \mathrm{H}$ NMR and ${ }^{13} \mathrm{C}$ NMR spectra were recorded on a Varian-300 spectrometer using TMS as an internal reference. Mass spectra (GC-MS) were obtained on an Agilest 68905973 instrument. Elemental analysis was performed on a Yananca CDRDER MT-3A elemental analyzer (The analysis was carried out by state key laboratory of Elemeto-Organic chemistry, Nankai University, Tianjin 30071, P.R.China). The structures of new compounds were confirmed by ${ }^{1} \mathrm{H}$ NMR and elemental analysis (Table 2 and Table 3). Some compound structures were further confirmed by ${ }^{13} \mathrm{C}$ NMR and mass spectra (Table 4). 
Table 1. Herbicidal activity

\begin{tabular}{lcccc|lcccc}
\hline Comd & $\begin{array}{c}\text { Dose } \\
(\mathrm{g} / \mathrm{ha})\end{array}$ & BYG & CRB & VEL & Comd & $\begin{array}{c}\text { Dose } \\
(\mathrm{g} / \mathrm{ha})\end{array}$ & BYG & CRB & VEL \\
\hline $2 \mathrm{a}$ & 1000 & 0 & 0 & 10 & $2 \mathrm{~b}$ & 1000 & 0 & 10 & 95 \\
& 200 & 0 & 0 & 0 & & 200 & 0 & 0 & 0 \\
$\mathrm{2c}$ & 1000 & 0 & 0 & 0 & $2 \mathrm{~d}$ & 1000 & 0 & 0 & 10 \\
& 200 & 0 & 0 & 0 & & 200 & 0 & 0 & 0 \\
$3 \mathrm{a}$ & 1000 & 10 & 35 & 70 & $3 \mathrm{~b}$ & 1000 & 10 & 50 & 98 \\
& 200 & 0 & 15 & 20 & & 200 & 0 & 0 & 0 \\
$3 \mathrm{c}$ & 1000 & 50 & 70 & 100 & $3 \mathrm{~d}$ & 1000 & 50 & 70 & 100 \\
& 200 & 0 & 10 & 95 & & 200 & 10 & 40 & 90 \\
$3 \mathrm{e}$ & 1000 & 70 & 70 & 100 & $3 \mathrm{f}$ & 1000 & 5 & 15 & 95 \\
& 200 & 20 & 20 & 100 & & 200 & 5 & 10 & 90 \\
$3 \mathrm{~g}$ & 1000 & 0 & 0 & 45 & $3 \mathrm{~h}$ & 1000 & 0 & 0 & 85 \\
& 200 & 0 & 0 & 30 & & 200 & 0 & 0 & 75 \\
$3 \mathrm{i}$ & 1000 & 0 & 0 & 30 & $3 \mathrm{j}$ & 1000 & 5 & 5 & 30 \\
& 200 & 0 & 0 & 10 & & 200 & 5 & 5 & 25 \\
$4 \mathrm{a}$ & 1000 & 0 & 10 & 95 & $4 \mathrm{~b}$ & 1000 & 5 & 5 & 10 \\
& 200 & 0 & 0 & 0 & & 200 & 0 & 0 & 0 \\
$4 \mathrm{c}$ & 1000 & 5 & 10 & 25 & $4 \mathrm{~d}$ & 1000 & 40 & 35 & 80 \\
& 200 & 0 & 0 & 0 & & 200 & 30 & 20 & 55 \\
$5 \mathrm{a}$ & 1000 & 50 & 70 & 75 & $5 \mathrm{~b}$ & 1000 & 0 & 0 & 0 \\
$5 \mathrm{c}$ & 1000 & 0 & 0 & 0 & $5 \mathrm{~d}$ & 1000 & 5 & 0 & 20 \\
$5 \mathrm{e}$ & 1000 & 10 & 5 & 10 & $5 \mathrm{f}$ & 1000 & 0 & 30 & 60 \\
\hline
\end{tabular}

0 equals no activity; 100 equals total control. BYG: Barnyard (Echinochloa crusgalli); CRB: crabgrass (Digitaria sanguinalis). VEL: Velvetleaf (Abutilon thophrasti).

2-(6-(2-Chloro-4-trifluoromethyl)phenoxy)-2,4-dioxo-1,2-dihydroquinazolin-3(4H)-yl) acetates (2). A mixture of 1 (1 $1 \mathrm{mmol})$, triphosgene $(1.2 \mathrm{mmol}), \mathrm{Et}_{3} \mathrm{~N}(2.4 \mathrm{mmol})$ was stirred in $\mathrm{CH}_{2} \mathrm{Cl}_{2}$ on an ice-water bath for $2 \mathrm{~h}$. The reaction mixture was extracted with $\mathrm{CH}_{2} \mathrm{Cl}_{2}(2 \times 100 \mathrm{~mL})$, washed with the solution of $\mathrm{NaHCO}_{3}, \mathrm{NaCl}$, dried over anhydrous magnesium sulfate and the solvent evaporated. The residue was purified by column chromatography $(20 \%$ EtOAc in hexanes as eluent) to give the target compound.

Ethyl 2-(6-(2-chloro-4-(trifluoromethyl)phenoxy)-1,2-dihydro-2,4-dioxoquinazolin-3(4H)yl) acetate (2b). Yield $75 \%$, white solid, mp 199-201 ${ }^{\circ} \mathrm{C} .{ }^{1} \mathrm{H}$ NMR $\left(300 \mathrm{MHz}, \mathrm{CDCl}_{3}\right) \delta(\mathrm{ppm})$ $1.30(\mathrm{t}, J 6.9 \mathrm{~Hz}, 3 \mathrm{H}), 4.26$ (q, $J 6.9 \mathrm{~Hz}, 2 \mathrm{H}), 4.83(\mathrm{~s}, 2 \mathrm{H}), 7.01$ (d, $J 8.7 \mathrm{~Hz}, 1 \mathrm{H}, \mathrm{Ar}-\mathrm{H}), 7.21$ (d, $J 8.7 \mathrm{~Hz}, 1 \mathrm{H}, \mathrm{Ar}-\mathrm{H}), 7.39$ (dd, $J 3.0$ and $3.0 \mathrm{~Hz}, 1 \mathrm{H}, \mathrm{Ar}-\mathrm{H}$ ), 7.48 (dd, $J 2.1$ and $3.0 \mathrm{~Hz}, 1 \mathrm{H}, \mathrm{Ar}-$ H), 7.64 (d, 1H, J $3.0 \mathrm{~Hz}, \mathrm{Ar}-\mathrm{H}), 7.75$ (d, 1H, J 2.1 Hz, Ar-H), 10.79 (s, 1H, H-NCO). Anal. Calcd. for $\mathrm{C}_{19} \mathrm{H}_{14} \mathrm{ClF}_{3} \mathrm{~N}_{2} \mathrm{O}_{5}$ : C, 51.54; H, 3.19; N, 6.33; Found: C, 51.6; H, 3.44; N, 6.19. 
Table 2. Physical constants and elemental analysis

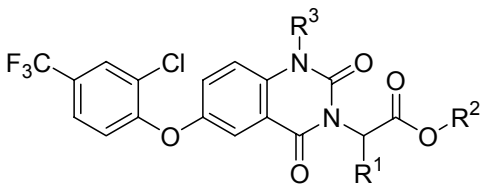

\begin{tabular}{|c|c|c|c|c|c|c|c|c|}
\hline \multirow{2}{*}{ Cpd } & \multirow{2}{*}{$\mathrm{R}^{1}$} & \multirow{2}{*}{$\mathrm{R}^{2}$} & \multirow{2}{*}{$\mathrm{R}^{3}$} & \multirow{2}{*}{ Yield, (\%) } & \multirow{2}{*}{ M.p. $\left({ }^{\circ} \mathrm{C}\right)$} & \multicolumn{3}{|c|}{ Analysis $(\%),($ calc./found $)$} \\
\hline & & & & & & $\mathrm{C}$ & $\mathrm{H}$ & $\mathrm{N}$ \\
\hline \multirow{2}{*}{$2 \mathrm{a}$} & \multirow{2}{*}{$\mathrm{H}$} & \multirow{2}{*}{$\mathrm{Me}$} & \multirow{2}{*}{$\mathrm{H}$} & \multirow{2}{*}{71} & \multirow{2}{*}{$202-204$} & 50.42 & 2.82 & 6.53 \\
\hline & & & & & & 50.31 & 3.01 & 6.41 \\
\hline \multirow{2}{*}{$2 b$} & \multirow{2}{*}{$\mathrm{H}$} & \multirow{2}{*}{ ethyl } & \multirow{2}{*}{$\mathrm{H}$} & \multirow{2}{*}{75} & \multirow{2}{*}{ 199-201 } & 51.54 & 3.19 & 6.33 \\
\hline & & & & & & 51.66 & 3.34 & 6.19 \\
\hline \multirow{2}{*}{$2 \mathrm{c}(R)$} & \multirow{2}{*}{$\mathrm{Me}$} & \multirow{2}{*}{$\mathrm{Me}$} & \multirow{2}{*}{$\mathrm{H}$} & \multirow{2}{*}{67} & \multirow{2}{*}{$32-134$} & 51.54 & 3.19 & 6.33 \\
\hline & & & & & & 51.40 & 3.01 & 6.03 \\
\hline \multirow{2}{*}{$2 \mathrm{~d}(S)$} & Me & Me & $\mathrm{H}$ & 70 & 110 & 51.54 & 3.19 & 6.33 \\
\hline & Me & Me & $\mathrm{H}$ & 10 & $119-121$ & 51.78 & 3.09 & 6.08 \\
\hline $3 a$ & $\mathrm{H}$ & $\mathrm{Me}$ & $\mathrm{Me}$ & 65 & 190-192 & 51.54 & 3.19 & 6.33 \\
\hline & & & & & $190-192$ & 51.31 & 3.22 & 6.60 \\
\hline $3 \mathrm{~h}$ & $\mathrm{H}$ & ethyl & Мe & 63 & בריב-201 & 52.59 & 3.53 & 6.13 \\
\hline 50 & $\mathrm{H}$ & ethyl & Me & 63 & $201-202$ & 52.78 & 3.66 & 6.58 \\
\hline $3 c$ & $\mathrm{H}$ & $\mathrm{Me}$ & allyl & 65 & yellow & 53.80 & 3.44 & 5.98 \\
\hline & & & & & oil & 53.63 & 3.24 & 5.80 \\
\hline $3 d$ & $\mathrm{H}$ & ethyl & allyl & 63 & 120-120 & 54.03 & 3.76 & 5.80 \\
\hline $5 \mathrm{a}$ & $\mathrm{H}$ & etnys & anys & 03 & $120-1<2$ & 54.68 & 3.78 & 5.62 \\
\hline $3 e$ & $\mathrm{H}$ & Me & nronyny1 & 66 & yellow & 54.03 & 3.02 & 6.00 \\
\hline$J$ & $\Pi$ & Mie & propyny1 & 06 & oil & 53.89 & 2.98 & 6.13 \\
\hline $3 f$ & $\mathrm{H}$ & ethvl & nronynvl & 64 & $118-120$ & 54.96 & 3.35 & 5.83 \\
\hline & & etnyl & propynyı & 04 & & 54.87 & 3.37 & 5.80 \\
\hline $3 \alpha(P)$ & Mo & Me & olly1 & 61 & 130121 & 54.73 & 3.76 & 5.80 \\
\hline $\operatorname{sg}(K)$ & IVIe & Me & allyı & 01 & $150-131$ & 54.99 & 3.85 & 5.49 \\
\hline $3 \mathrm{~h}(\Omega$ & Me & Me & allyl & 64 & yellow & 54.73 & 3.76 & 5.80 \\
\hline $\operatorname{sn}(2)$ & IVIe & Me & allyı & 04 & oil & 54.60 & 3.81 & 5.88 \\
\hline $3 \mathrm{I}(R)$ & $\mathrm{Me}$ & $\mathrm{Me}$ & pronynyl & 62 & $118-119$ & 54.96 & 3.35 & 5.83 \\
\hline & & & & 02 & & 54.76 & 3.76 & 5.80 \\
\hline $3 \mathrm{i}(S)$ & $\mathrm{Me}$ & $\mathrm{Me}$ & pronynyl & 60 & yellow & 54.96 & 3.35 & 5.83 \\
\hline & & Nie & ргорупу1 & 60 & oil & 55.20 & 3.69 & 6.00 \\
\hline$A_{0}$ & $\mathrm{H}$ & $\mathrm{H}$ & $\mathrm{H}$ & 55 & $100-10 ?$ & 49.23 & 2.43 & 6.75 \\
\hline $4 a$ & Н & $\Pi$ & $\Pi$ & 55 & $190-192$ & 49.02 & $2 . .78$ & 6.45 \\
\hline $4 \mathrm{~h}$ & $\mathrm{H}$ & $\mathrm{H}$ & Me & 58 & $100-106$ & 50.42 & 2.82 & 6.53 \\
\hline & & & IVIe & 30 & 190 & 50.39 & 2.89 & 6.40 \\
\hline $4 c$ & $\mathrm{H}$ & $\mathrm{H}$ & allyl & 60 & $172-174$ & 52.82 & 3.10 & 6.16 \\
\hline & 11 & 11 & allyı & 00 & $1 / 2-1 / 4$ & 52.48 & 3.26 & 6.59 \\
\hline $4 d$ & $\mathrm{H}$ & $\mathrm{H}$ & pronynyl & 60 & $200-202$ & 53.05 & 2.67 & 6.19 \\
\hline & & H & ргорупу1 & 00 & $200-202$ & 53.44 & 2.88 & 6.09 \\
\hline
\end{tabular}




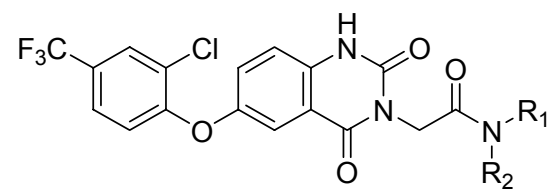

\begin{tabular}{|c|c|c|c|c|c|c|c|}
\hline \multirow[t]{2}{*}{ Cpd } & \multirow[t]{2}{*}{$\mathrm{R}^{1}$} & \multirow[t]{2}{*}{$\mathrm{R}^{2}$} & \multirow[t]{2}{*}{ Yield (\%) } & \multirow[t]{2}{*}{$\mathrm{mp}\left({ }^{\circ} \mathrm{C}\right)$} & \multicolumn{3}{|c|}{$\begin{array}{l}\text { Analysis (\%) } \\
\text { (calc./found) }\end{array}$} \\
\hline & & & & & $\mathrm{C}$ & $\mathrm{H}$ & $\mathrm{N}$ \\
\hline \multirow{2}{*}{$5 \mathrm{a}$} & \multirow{2}{*}{$\mathrm{H}$} & \multirow{2}{*}{$\mathrm{Me}$} & \multirow{2}{*}{54} & \multirow{2}{*}{$238-241$} & 50.54 & 3.06 & 9.82 \\
\hline & & & & & 50.66 & 3.25 & 9.99 \\
\hline \multirow{2}{*}{$5 b$} & \multirow{2}{*}{$\mathrm{H}$} & \multirow{2}{*}{ isopropyl } & \multirow{2}{*}{56} & \multirow{2}{*}{$252-254$} & 52.70 & 3.76 & 9.22 \\
\hline & & & & & 52.52 & 4.00 & 9.12 \\
\hline \multirow{2}{*}{$5 c$} & \multirow{2}{*}{$\mathrm{H}$} & \multirow{2}{*}{ phenyl } & \multirow{2}{*}{51} & \multirow{2}{*}{$270-272$} & 56.40 & 3.09 & 8.58 \\
\hline & & & & & 56.81 & 3.39 & 8.79 \\
\hline \multirow{2}{*}{$5 \mathrm{~d}$} & \multirow{2}{*}{$\mathrm{H}$} & \multirow{2}{*}{ p-tolyl } & \multirow{2}{*}{52} & \multirow{2}{*}{$280-281$} & 57.21 & 3.40 & 8.34 \\
\hline & & & & & 57.11 & 3.33 & 8.31 \\
\hline \multirow{2}{*}{$5 e$} & \multirow{2}{*}{$\mathrm{H}$} & \multirow{2}{*}{ 4-nitrophenyl } & \multirow{2}{*}{52} & \multirow{2}{*}{$247-248$} & 51.65 & 2.64 & 10.68 \\
\hline & & & & & 51.70 & 2.45 & 10.99 \\
\hline \multirow{2}{*}{$5 \mathrm{f}$} & \multirow{2}{*}{\multicolumn{2}{|c|}{ morpholin }} & \multirow{2}{*}{50} & \multirow{2}{*}{$239-241$} & 52.13 & 3.54 & 8.68 \\
\hline & & & & & 52.09 & 3.13 & 8.45 \\
\hline
\end{tabular}

Table 3. ${ }^{1} \mathrm{H}$ NMR

\begin{tabular}{cl}
\hline Cpd & ${ }^{1} \mathrm{H} \mathrm{NMR}\left(300 \mathrm{MHz}, \mathrm{CDCl}_{3}\right) \delta(\mathrm{ppm})$ \\
\hline 2a & $3.80(\mathrm{~s}, 3 \mathrm{H}), 4.85(\mathrm{~s}, 2 \mathrm{H}), 7.02(\mathrm{~d}, J 9.0 \mathrm{~Hz}, 1 \mathrm{H}, \mathrm{Ar}-\mathrm{H}), 7.22(\mathrm{~d}, J 9.0 \mathrm{~Hz}, 1 \mathrm{H}, \mathrm{Ar}-\mathrm{H})$, \\
& $7.41(\mathrm{dd}, J 3.0$ and $3.0 \mathrm{~Hz}, 1 \mathrm{H}, \mathrm{Ar}-\mathrm{H}), 7.49(\mathrm{dd}, J 2.1$ and $3.0 \mathrm{~Hz}, 1 \mathrm{H}, \mathrm{Ar}-\mathrm{H}), 7.65(\mathrm{~d}$, \\
& $1 \mathrm{H}, J 3.0 \mathrm{~Hz}, \mathrm{Ar}-\mathrm{H}), 7.77(\mathrm{~d}, 1 \mathrm{H}, J 2.1 \mathrm{~Hz}, \mathrm{Ar}-\mathrm{H}), 10.66(\mathrm{~s}, 1 \mathrm{H}, \mathrm{H}-\mathrm{NCO})$ \\
2b & $1.30(\mathrm{t}, J 6.9 \mathrm{~Hz}, 3 \mathrm{H}), 4.26(\mathrm{q}, J 6.9 \mathrm{~Hz}, 2 \mathrm{H}), 4.83(\mathrm{~s}, 2 \mathrm{H}), 7.01(\mathrm{~d}, J 8.7 \mathrm{~Hz}, 1 \mathrm{H}, \mathrm{Ar}-$ \\
& $\mathrm{H}), 7.21(\mathrm{~d}, J 8.7 \mathrm{~Hz}, 1 \mathrm{H}, \mathrm{Ar}-\mathrm{H}), 7.39(\mathrm{dd}, J 3.0$ and $3.0 \mathrm{~Hz}, 1 \mathrm{H}, \mathrm{Ar}-\mathrm{H}), 7.48(\mathrm{dd}, J 2.1$ \\
& and $3.0 \mathrm{~Hz}, 1 \mathrm{H}, \mathrm{Ar}-\mathrm{H}), 7.64(\mathrm{~d}, 1 \mathrm{H}, J 3.0 \mathrm{~Hz}, \mathrm{Ar}-\mathrm{H}), 7.75(\mathrm{~d}, 1 \mathrm{H}, J 2.1 \mathrm{~Hz}, \mathrm{Ar}-\mathrm{H})$, \\
& $10.79(\mathrm{~s}, 1 \mathrm{H}, \mathrm{H}-\mathrm{NCO})$ \\
2c $(\mathrm{R})$ & $1.67\left(\mathrm{~d}, 3 \mathrm{H}, J 6.9 \mathrm{~Hz}, \mathrm{CH}_{3}\right), 3.75\left(\mathrm{~s}, 3 \mathrm{H}, \mathrm{OCH}_{3}\right), 5.64(\mathrm{q}, 1 \mathrm{H}, J 6.9 \mathrm{~Hz}, \mathrm{CH}), 7.03(\mathrm{~d}$, \\
& $1 \mathrm{H}, J 8.4 \mathrm{~Hz}, \mathrm{Ar}-\mathrm{H}), 7.17(\mathrm{~d}, 1 \mathrm{H}, J 8.4 \mathrm{~Hz}, \mathrm{Ar}-\mathrm{H}), 7.42(\mathrm{dd}, 1 \mathrm{H}, J 2.7 \mathrm{and} 8.7 \mathrm{~Hz}, \mathrm{Ar}-$ \\
& $\mathrm{H}), 7.50(\mathrm{dd}, 1 \mathrm{H}, J 2.4 \mathrm{and} 6.0 \mathrm{~Hz}, \mathrm{Ar}-\mathrm{H}), 7.64(\mathrm{~d}, 1 \mathrm{H}, J 2.7 \mathrm{~Hz}, \mathrm{Ar}-\mathrm{H}), 7.77(\mathrm{~d}, 1 \mathrm{H}, J$ \\
& $1.5 \mathrm{~Hz}, \mathrm{Ar}-\mathrm{H})$ \\
2d $(\mathrm{S}) \quad$ & $1.68(\mathrm{~d}, 3 \mathrm{H}, J 6.9 \mathrm{~Hz}, \mathrm{CH}), 3.75(\mathrm{~s}, 3 \mathrm{H}, \mathrm{OCH}), 5.65(\mathrm{q}, 1 \mathrm{H}, J 6.9 \mathrm{~Hz}, \mathrm{CH}), 7.03(\mathrm{~d}$, \\
& $1 \mathrm{H}, J 8.4 \mathrm{~Hz}, \mathrm{Ar}-\mathrm{H}), 7.19(\mathrm{~d}, 1 \mathrm{H}, J 9.0 \mathrm{~Hz}, \mathrm{Ar}-\mathrm{H}), 7.42(\mathrm{dd}, 1 \mathrm{H}, J 3.0 \mathrm{and} 8.7 \mathrm{~Hz}, \mathrm{Ar}-$ \\
& $\mathrm{H}), 7.50(\mathrm{dd}, 1 \mathrm{H}, J 2.4 \mathrm{and} 6.0 \mathrm{~Hz}, \mathrm{Ar}-\mathrm{H}), 7.64(\mathrm{~d}, 1 \mathrm{H}, J 2.7 \mathrm{~Hz}, \mathrm{Ar}-\mathrm{H}), 7.77(\mathrm{~d}, 1 \mathrm{H}, J$ \\
& $1.8 \mathrm{~Hz}, \mathrm{Ar}-\mathrm{H})$ \\
& $3.64(\mathrm{~s}, 3 \mathrm{H}), 3.78(\mathrm{~s}, 3 \mathrm{H}), 4.85(\mathrm{~s}, 2 \mathrm{H}), 7.17(\mathrm{~d}, J 9 \mathrm{~Hz}, 1 \mathrm{H}, \mathrm{Ar}-\mathrm{H}), 7.61-7.69(\mathrm{~m}, 3 \mathrm{H}$, \\
& $\mathrm{Ar}-\mathrm{H}), 7.70(\mathrm{~d}, J 9 \mathrm{~Hz}, 1 \mathrm{H}, \mathrm{Ar}-\mathrm{H}), 7.98(\mathrm{~s}, 1 \mathrm{H}, \mathrm{Ar}-\mathrm{H})$
\end{tabular}


3b $\quad 1.23(\mathrm{t}, J 6.9 \mathrm{~Hz}, 3 \mathrm{H}), 3.57(\mathrm{~s}, 3 \mathrm{H}), 4.15(\mathrm{q}, J 6.9 \mathrm{~Hz}, 2 \mathrm{H}), 4.67(\mathrm{~s}, 2 \mathrm{H}), 7.17$ (d, $J 9.0$ $\mathrm{Hz}, 1 \mathrm{H}, \mathrm{Ar}-\mathrm{H}), 7.61-7.69$ (m, 3H, Ar-H), 7.70(d, J9.0 Hz, 1H, Ar-H), 7.98 (s, $1 \mathrm{H}, \mathrm{Ar}-$ $\mathrm{H})$

3c $\quad 3.78(\mathrm{~s}, 3 \mathrm{H}), 4.79(\mathrm{~d}, J 2.1 \mathrm{~Hz}, 2 \mathrm{H}), 4.83(\mathrm{~s}, 2 \mathrm{H}), 5.29-5.31(\mathrm{~m}, 2 \mathrm{H}), 5.88-5.92(\mathrm{~m}$, 1H), $7.02(\mathrm{~d}, J 8.1 \mathrm{~Hz}, 1 \mathrm{H}, \mathrm{Ar}-\mathrm{H}), 7.23(\mathrm{~s}, 1 \mathrm{H}, \mathrm{Ar}-\mathrm{H}), 7.41(\mathrm{~d}, J 8.1 \mathrm{~Hz}, 1 \mathrm{H}, \mathrm{Ar}-\mathrm{H})$, 7.49 (d, $J 8.1 \mathrm{~Hz}, 1 \mathrm{H}, \mathrm{Ar}-\mathrm{H}), 7.76$ (d, 2H, Ar-H)

3d $\quad 1.29(\mathrm{t}, J 6.9 \mathrm{~Hz}, 3 \mathrm{H}), 4.23(\mathrm{q}, J 6.9 \mathrm{~Hz}, 2 \mathrm{H}), 4.82(\mathrm{~s}, 2 \mathrm{H}), 5.26-5.31(\mathrm{~m}, 2 \mathrm{H}), 5.89-$ 5.98 (m, 1H), 7.03 (d, J $9.0 \mathrm{~Hz}, 1 \mathrm{H}, \mathrm{Ar}-\mathrm{H}), 7.26$ (d, J 9.0 Hz, 1H, Ar-H), 7.39-7.51 (m, 2H, Ar-H), 7.760 (s, 2H, Ar-H)

3e $\quad 2.35(\mathrm{t}, J 2.4 \mathrm{~Hz}, 1 \mathrm{H}), 3.78(\mathrm{~s}, 3 \mathrm{H}), 4.84(\mathrm{~s}, 2 \mathrm{H}), 4.94(\mathrm{~d}, J 2.4 \mathrm{~Hz}, 2 \mathrm{H}), 7.03(\mathrm{~d}, J 8.4$ $\mathrm{Hz}, 1 \mathrm{H}, \mathrm{Ar}-\mathrm{H})$, 7.47-7.51 (m, 3H, Ar-H), 7.75-7.77 (m, 2H, Ar-H)

ff $\quad 1.29(\mathrm{t}, J 6.9 \mathrm{~Hz}, 3 \mathrm{H}), 2.35(\mathrm{q}, J 3.1 \mathrm{~Hz}, 1 \mathrm{H}), 4.25(\mathrm{q}, J 6.9 \mathrm{~Hz}, 2 \mathrm{H}), 4.78(\mathrm{~s}, 2 \mathrm{H}), 4.95$ (s, 2H), $7.03(\mathrm{~d}, J 9.0 \mathrm{~Hz}, 1 \mathrm{H}, \mathrm{Ar}-\mathrm{H}), 7.64-7.78(\mathrm{~m}, 4 \mathrm{H}, \mathrm{Ar}-\mathrm{H}), 7.77$ (s, 1H, Ar-H)

$3 \mathrm{~g}(R) \quad 1.64\left(\mathrm{~d}, 3 \mathrm{H}, J 6.9 \mathrm{~Hz}, \mathrm{CH}_{3}\right), 3.75\left(\mathrm{~s}, 3 \mathrm{H}, \mathrm{OCH}_{3}\right), 4.77\left(\mathrm{t}, 2 \mathrm{H}, J 6.9 \mathrm{~Hz}, \mathrm{CH}_{2}\right), 5.29-5.33$ (m, 2H, C CH), $5.64(\mathrm{q}, J 6.9 \mathrm{~Hz}, 1 \mathrm{H}, \mathrm{CH}), 5.89-5.98(\mathrm{~m}, 1 \mathrm{H},-\mathrm{CH}), 7.03(\mathrm{~d}, 1 \mathrm{H}, J$ $8.7 \mathrm{~Hz}, \mathrm{Ar}-\mathrm{H}), 7.25$ (d, $1 \mathrm{H}, J 9.0 \mathrm{~Hz}, \mathrm{Ar}-\mathrm{H}), 7.44$ (dd, $1 \mathrm{H}, J 3.0$ and $6.0 \mathrm{~Hz}, \mathrm{Ar}-\mathrm{H})$, 7.49 (dd, 1H, J 2.4 and $8.7 \mathrm{~Hz}$, Ar-H), 7.74 (d, 1H, J 3.0 Hz, Ar-H), 7.77 (d, 1H, J 2.1 $\mathrm{Hz}, \mathrm{Ar}-\mathrm{H})$

3h (S) $\quad 1.65$ (d, 3H, J 6.9 Hz, $\left.\mathrm{CH}_{3}\right), 3.73\left(\mathrm{~s}, 3 \mathrm{H}, \mathrm{OCH}_{3}\right), 4.78$ (t, $\left.2 \mathrm{H}, J 6.9 \mathrm{~Hz}, \mathrm{CH}_{2}\right), 5.29-5.32$ (m, 2H, C CH), 5.64 (q, 1H, J 6.3 Hz, CH), 5.93-5.99 (m, 1H, -CH ), 7.04 (d, 1H, $J$ $8.7 \mathrm{~Hz}, \mathrm{Ar}-\mathrm{H}), 7.23(\mathrm{~d}, 1 \mathrm{H}, J 9.0 \mathrm{~Hz}, \mathrm{Ar}-\mathrm{H}), 7.42(\mathrm{dd}, 1 \mathrm{H}, J 3.0$ and $6.0 \mathrm{~Hz}, \mathrm{Ar}-\mathrm{H})$, 7.48 (dd, $1 \mathrm{H}, J 2.4$ and $8.7 \mathrm{~Hz}, \mathrm{Ar}-\mathrm{H}), 7.74$ (d, 1H, J 3.0 Hz, Ar-H), 7.76 (d, 1H, $J$ $2.1 \mathrm{~Hz}, \mathrm{Ar}-\mathrm{H})$

$3 \mathrm{i}(R) \quad 1.64\left(\mathrm{~d}, 3 \mathrm{H}, J 6.9 \mathrm{~Hz}, \mathrm{CH}_{3}\right), 2.37$ (t, $\left.1 \mathrm{H}, J 3.1 \mathrm{~Hz}, \mathrm{C} \equiv \mathrm{H}\right), 3.74\left(\mathrm{~s}, 3 \mathrm{H}, \mathrm{OCH}_{3}\right), 4.94$ (d, 2H, J $3.1 \mathrm{~Hz}, \mathrm{CH}_{2}$ ), 5.63 (q, 1H, J 6.9 Hz, CH), 7.05 (d, 1H, J 8.4 Hz, Ar-H), 7.49 (m, $3 \mathrm{H}, \mathrm{Ar}-\mathrm{H}), 7.74(\mathrm{~d}, 1 \mathrm{H}, J 1.8 \mathrm{~Hz}, \mathrm{Ar}-\mathrm{H}), 7.77$ (d, $1 \mathrm{H}, J 1.8 \mathrm{~Hz}, \mathrm{Ar}-\mathrm{H})$

$3 \mathrm{j}(S) \quad 1.64\left(\mathrm{~d}, 3 \mathrm{H}, J 6.9 \mathrm{~Hz}, \mathrm{CH}_{3}\right), 2.36(\mathrm{t}, 1 \mathrm{H}, J 3.0 \mathrm{~Hz}, \mathrm{C} \equiv \mathrm{CH}), 3.74\left(\mathrm{~s}, 3 \mathrm{H}, \mathrm{OCH}_{3}\right), 4.95(\mathrm{t}$, $\left.2 \mathrm{H}, J 3.0 \mathrm{~Hz}, \mathrm{CH}_{2}\right), 5.62(\mathrm{q}, 1 \mathrm{H}, J 6.9 \mathrm{~Hz}, \mathrm{CH}), 7.06$ (d, $\left.1 \mathrm{H}, J 8.4 \mathrm{~Hz}, \mathrm{Ar}-\mathrm{H}\right), 7.51(\mathrm{~m}$, $3 \mathrm{H}, \mathrm{Ar}-\mathrm{H}), 7.76$ (d, $1 \mathrm{H}, J 8.4 \mathrm{~Hz}, \mathrm{Ar}-\mathrm{H}), 7.78$ (d, $1 \mathrm{H}, J 1.8 \mathrm{~Hz}, \mathrm{Ar}-\mathrm{H})$

4a $\quad 4.54(\mathrm{~s}, 2 \mathrm{H}), 7.04(\mathrm{~d}, J 9.0 \mathrm{~Hz}, 1 \mathrm{H}, \mathrm{Ar}-\mathrm{H}), 7.31(\mathrm{~d}, J 9.0 \mathrm{~Hz}, 1 \mathrm{H}, \mathrm{Ar}-\mathrm{H}), 7.40(\mathrm{dd}, J 3.0$ and $9.0 \mathrm{~Hz}, 1 \mathrm{H}, \mathrm{Ar}-\mathrm{H}), 7.43(\mathrm{dd}, J 2.1$ and $3.0 \mathrm{~Hz}, 1 \mathrm{H}, \mathrm{Ar}-\mathrm{H}), 7.59$ (d, $1 \mathrm{H}, J 3.0 \mathrm{~Hz}$, Ar-H), 7.81 (d, 1H, J 2.1 Hz, Ar-H), 11.69 (s, 1H, COOH)

4b $\quad 3.58(\mathrm{~s}, 3 \mathrm{H}), 4.60(\mathrm{~s}, 2 \mathrm{H}), 7.15(\mathrm{~d}, 1 \mathrm{H}, J 8.1 \mathrm{~Hz}$ Ar-H), 7.60-7.67 (m, 4H, Ar-H), 7.93 $(\mathrm{s}, 1 \mathrm{H})$

4c $\quad 4.61(\mathrm{~s}, 2 \mathrm{H}), 4.80(\mathrm{~d}, J 3.1 \mathrm{~Hz}, 2 \mathrm{H}), 5.22(\mathrm{t}, J 3.1 \mathrm{~Hz}, 2 \mathrm{H}), 5.93(\mathrm{~m}, 1 \mathrm{H}), 7.15(\mathrm{~d}, J 8.4$ $\mathrm{Hz}, 1 \mathrm{H}, \mathrm{Ar}-\mathrm{H}), 7.51(\mathrm{~m}, 2 \mathrm{H}, \mathrm{Ar}-\mathrm{H}), 7.65(\mathrm{~m}, 2 \mathrm{H}, \mathrm{Ar}-\mathrm{H}), 7.88(\mathrm{~d}, J 8.4 \mathrm{~Hz}, 1 \mathrm{H}, \mathrm{Ar}-\mathrm{H})$

4d $\quad 2.25(\mathrm{t}, J 2.4 \mathrm{~Hz}, 1 \mathrm{H}), 4.82(\mathrm{~s}, 2 \mathrm{H}), 4.96(\mathrm{~d}, J 2.4 \mathrm{~Hz}, 2 \mathrm{H}), 7.13(\mathrm{~d}, J 8.4 \mathrm{~Hz}, 1 \mathrm{H}$, Ar$\mathrm{H})$, 7.56-7.62 (m, 3H, Ar-H), 7.77-7.88 (m, 2H, Ar-H)

5a $2.63(\mathrm{~s}, 3 \mathrm{H}), 4.47(\mathrm{~s}, 2 \mathrm{H}), 7.06(\mathrm{~d}, J 8.7 \mathrm{~Hz}, 1 \mathrm{H}, \mathrm{Ar}-\mathrm{H}), 7.19-7.59(\mathrm{~m}, 4 \mathrm{H}, \mathrm{Ar}-\mathrm{H}), 7.86$ (s, $1 \mathrm{H}, \mathrm{Ar}-\mathrm{H})$ 
$5 \mathrm{~b} \quad 1.08(\mathrm{t}, J 6 \mathrm{~Hz}, 6 \mathrm{H}), 4.01(\mathrm{~m}, 1 \mathrm{H}), 4.41(\mathrm{~s}, 2 \mathrm{H}), 7.33(\mathrm{~d}, J 8.4 \mathrm{~Hz}, 1 \mathrm{H}, \mathrm{Ar}-\mathrm{H}), 7.33-$ $7.80(\mathrm{~m}, 4 \mathrm{H}, \mathrm{Ar}-\mathrm{H}), 7.96(\mathrm{~s}, 1 \mathrm{H}, \mathrm{Ar}-\mathrm{H})$

5c $\quad 4.69(\mathrm{~s}, 2 \mathrm{H}), 7.20(\mathrm{~m}, 1 \mathrm{H}, \mathrm{Ar}-\mathrm{H}), 7.11(\mathrm{~d}, J 9.0 \mathrm{~Hz}, 1 \mathrm{H}, \mathrm{Ar}-\mathrm{H}), 7.27(\mathrm{t}, J 9.0 \mathrm{~Hz}, 2 \mathrm{H}$, Ar-H), 7.35 (s, 1H, Ar-H), 7.48-7.65 (m, 5H, Ar-H), 7.90 (s, 1H, Ar-H), 10.20 (s, 1H), $11.69(\mathrm{~s}, 1 \mathrm{H})$

5d $2.27(\mathrm{~s}, 3 \mathrm{H}), 4.69(\mathrm{~s}, 2 \mathrm{H}), 7.14(\mathrm{~d}, J 8.7 \mathrm{~Hz}, 1 \mathrm{H}, \mathrm{Ar}-\mathrm{H}), 7.30-7.54(\mathrm{~m}, 3 \mathrm{H}, \mathrm{Ar}-\mathrm{H})$, 7.56-7.64 (m, 4H, Ar-H), 7.67 (d, J 8.7 Hz, 1H, Ar-H), 7.84 (s, 1H, Ar-H), 10.40 (s, $1 \mathrm{H})$

5e $\quad 4.75(\mathrm{~s}, 2 \mathrm{H}), 7.11(\mathrm{~d}, J 8.4 \mathrm{~Hz}, 1 \mathrm{H}, \mathrm{Ar}-\mathrm{H}), 7.36(\mathrm{~d}, J 8.4 \mathrm{~Hz}, 1 \mathrm{H}, \mathrm{Ar}-\mathrm{H}), 7.45(\mathrm{t}, J 2.1$ $\mathrm{Hz}, 1 \mathrm{H}, \mathrm{Ar}-\mathrm{H}), 7.56$ (d, J 2.1 Hz, 1H, Ar-H), 7.59 (dd, J 2.4 Hz, 1H, Ar-H), 7.81-7.84 (m, 3H, Ar-H), 8.16 (d, J 8.4 Hz, 1H, Ar-H), 8.19 (s, 1H, Ar-H), 10.87 (s, 1H)

5f $\quad 3.19-3.66(\mathrm{~m}, 8 \mathrm{H}), 7.09(\mathrm{~d}, J 8.7 \mathrm{~Hz}, 1 \mathrm{H}, \mathrm{Ar}-\mathrm{H}), 7.32(\mathrm{~d}, J 8.7 \mathrm{~Hz}, 1 \mathrm{H}, \mathrm{Ar}-\mathrm{H}), 7.46$ (dd, J3.0 and $3.0 \mathrm{~Hz}, 1 \mathrm{H}, \mathrm{Ar}-\mathrm{H}), 7.52(\mathrm{dd}, J 2$ and $3 \mathrm{~Hz}, 1 \mathrm{H}, \mathrm{Ar}-\mathrm{H}), 7.62(\mathrm{~d}, 1 \mathrm{H}, J 3$ $\mathrm{Hz}, \mathrm{Ar}-\mathrm{H}), 7.88$ (d, 1H, J 2.1 Hz, Ar-H), 11.65 (s, 1H)

Table 4. ${ }^{13} \mathrm{C}$ NMR and MS data

\begin{tabular}{cl}
\hline Cpd & $13 \mathrm{C}$ NMR $(100 \mathrm{MHz}, \mathrm{CDCl} 3) \delta(\mathrm{ppm}) \mathrm{GC}-\mathrm{MS}(\mathrm{EI}), \mathrm{m} / \mathrm{z}(\%)$ \\
\hline $3 \mathrm{c}$ & $168.1,160.5,155.0,154.9,151.4,149.0,136.4,130.7,128.3,128.2,126.5,125.8$, \\
& $125.3,125.2,117.7,117.3,116.5,116.4,52.3,46.2,42.4$ \\
& $468(\mathrm{M}+, 100 \%), 437(19 \%), 408(32 \%), 380(21 \%), 352(86 \%), 340(98 \%), 213$ \\
& $(45 \%), 129(18 \%), 41(23 \%)$ \\
$3 \mathrm{~d}$ & $167.6,160.6,155.0,151.4,150.0,136.5,130.7,128.5,127.3,126.8,126.5,125.8$, \\
& $124.9,121.3,119.7,117.7,117.3,116.5,61.6,46.2,42.6,14.0$ \\
& $482(\mathrm{M}+, 82 \%), 437(19 \%), 408(38 \%), 380(19 \%), 352(69 \%), 340(100 \%), 213$ \\
& $(38 \%), 129(13 \%), 41(21 \%)$ \\
& $168.1,160.3,154.8,151.8,149.6,128.4,128.3,128.2,126.5,125.4,125.3,125.2$, \\
& $119.8,117.4,116.7,116.4,76.6,73.8,57.5,42.6,33.6$ \\
& $466(\mathrm{M}+, 100 \%), 435(7 \%), 406(53 \%), 378(8 \%), 364(7 \%), 350(67 \%), 323(20 \%)$, \\
& $288(17 \%), 179(10 \%), 156(24 \%), 128(12 \%), 56(10 \%)$ \\
& $167.6,160.4,151.8,149.7,135.6,128.4,128.3,126.5,126.0,125.4,125.3,119.8$, \\
$3 f$ & $117.4,116.7,116.5,116.4,76.7,73.7,67.0,42.8,33.6,14.0$ \\
& $480(\mathrm{M}+, 100 \%), 435(15 \%), 406(85 \%), 378(15 \%), 364(15 \%), 350(70 \%), 323$ \\
& $(15 \%), 288(14 \%), 179(14 \%), 156(25 \%), 128(15 \%), 56(13 \%)$.
\end{tabular}

2-(6-(2-Chloro-4-trifluoromethyl)phenoxy)-1,2-dihydro-1-alkyl-2,4-dioxoquinazolin-3(4H)yl)acetates (3). A mixture of 2 (1 $\mathrm{mmol}), \mathrm{K}_{2} \mathrm{CO}_{3}(1.2 \mathrm{mmol})$ and alkyl halide (1.1mmol) was stirred in $N, N$-dimethylformamide at $40{ }^{\circ} \mathrm{C}$. The reaction mixture was extracted with EtOAc $(2 \times 100 \mathrm{~mL})$, washed with a solution of $\mathrm{NaHCO}_{3}, \mathrm{NaCl}$, dried over anhydrous magnesium sulfate 
and the solvent evaporated. The residue was purified by column chromatography $(20 \%$ EtOAc in hexanes as eluent) to give the target compound.

Ethyl 2-(6-(2-chloro-4-(trifluoromethyl)phenoxy)-1,2-dihydro-1-propynyl-2,4-dioxoquinazolin-3-(4H)-yl) acetate (3f). Yield 64\%, white solid, mp 118-120 ${ }^{\circ} \mathrm{C}$. ${ }^{1} \mathrm{H}$ NMR $\left(300 \mathrm{MHz}, \mathrm{CDCl}_{3}\right) \delta(\mathrm{ppm}) 1.29(\mathrm{t}, J 6.9 \mathrm{~Hz}, 3 \mathrm{H}), 2.35(\mathrm{q}, J 3.1 \mathrm{~Hz}, 1 \mathrm{H}), 4.25(\mathrm{q}, J 6.9 \mathrm{~Hz}, 2 \mathrm{H})$, 4.78 (s, 2H), 4.95 (s, 2H), $7.03(\mathrm{~d}, J 9.0 \mathrm{~Hz}, 1 \mathrm{H}, \mathrm{Ar}-\mathrm{H}), 7.64-7.78(\mathrm{~m}, 4 \mathrm{H}, \mathrm{Ar}-\mathrm{H}), 7.77(\mathrm{~s}, 1 \mathrm{H}$, Ar-H). ${ }^{13} \mathrm{C}$ NMR $\left(100 \mathrm{MHz}, \mathrm{CDCl}_{3}\right) \delta(\mathrm{ppm}) 167.6,160.4,151.8,149.7,135.6,128.4,128.3$, $126.5,126.0,125.4,125.3,119.8,117.4,116.7,116.5,116.4,76.7,73.7,67.0,42.8,33.6,14.0$. GC-MS (EI), m/z (\%) $480\left(M^{+}, 100 \%\right), 435$ (15\%), 406 (85\%), 378 (15\%), 364 (15\%), 350 (70\%), 323 (15\%), 288 (14\%), 179 (14\%), 156 (25\%), 128 (15\%), 56 (13\%). Anal. Calcd. for $\mathrm{C}_{22} \mathrm{H}_{16} \mathrm{ClF}_{3} \mathrm{~N}_{2} \mathrm{O}_{5}$ : C, 54.96; H, 3.35; N, 5.83; Found: C, 54.87; H, 3.37; N, 5.80.

2-(6-(2-Chloro-4-(trifluoromethyl)phenoxy)-1,2-dihydro-2,4-dioxoquinazolin-3(4H)-yl) acetic acids (4). A mixture of $3(1 \mathrm{mmol})$ and aqueous $10 \% \mathrm{NaOH}(5 \mathrm{~mL})$ was refluxed in THF $(5 \mathrm{~mL})$ for $4 \mathrm{~h}$. After evaporation of the THF, $10 \% \mathrm{HCl}$ was added slowly to the residue under ice-water cooling. The resulting precipitates were collected by filtration, washed with water and recrystallized from EtOH to afford compounds 4.

2-(6-(2-Chloro-4-(trifluoromethyl)phenoxy)-1,2-dihydro-2,4-dioxoquinazolin-3(4H)-yl)

acetic acid (4a). Yield 55\%, white solid, mp 190-192 ${ }^{\circ} \mathrm{C} .{ }^{1} \mathrm{H} \mathrm{NMR}\left(300 \mathrm{MHz}, \mathrm{CDCl}_{3}\right) \delta(\mathrm{ppm})$ $4.54(\mathrm{~s}, 2 \mathrm{H}), 7.04$ (d, J9 Hz, 1H, Ar-H), 7.31 (d, J $9 \mathrm{~Hz}, 1 \mathrm{H}, \mathrm{Ar}-\mathrm{H}), 7.405$ (dd, $J 3.0$ and $3.0 \mathrm{~Hz}$, 1H, Ar-H), 7.43 (dd, J 2.1 and $3.0 \mathrm{~Hz}, 1 \mathrm{H}, \mathrm{Ar}-\mathrm{H}), 7.59$ (d, 1H, J 3.0 Hz, Ar-H), 7.81 (d, 1H, J 2 $\mathrm{Hz}, \mathrm{Ar}-\mathrm{H}), 11.69$ (s, $1 \mathrm{H}, \mathrm{COOH})$. Anal. Calcd. for $\mathrm{C}_{19} \mathrm{H}_{14} \mathrm{ClF}_{3} \mathrm{~N}_{2} \mathrm{O}_{5}$ : C, 49.23; $\mathrm{H}, 2.43 ; \mathrm{N}, 6.75$; Found: C, 49.02; H, 2.78; N, 6.45.

2-(6-(2-Chloro-4-trifluoromethyl)phenoxy)-2-(1-substituted-2,4dioxoquinazolin-3-(4H)-yl)$\mathrm{N}$-substituted acetamides (5). A mixture of 4 (1 $\mathrm{mmol})$ and oxalyl dichloride (2.0mmol) was stirred in $\mathrm{CH}_{2} \mathrm{Cl}_{2}$ at ice-water bath for $2 \mathrm{~h}$. Then the solvent was evaporated, afforded the yellow oil. The acyl chloride was added dropwise, the mixture of amide $(1 \mathrm{mmol})$, and $\mathrm{Et}_{3} \mathrm{~N}$ (1.1 mmol) was stirred in $\mathrm{CH}_{2} \mathrm{Cl}_{2}$ at room temperature for $2 \mathrm{~h}$. The reaction mixture was extracted with $\mathrm{CH}_{2} \mathrm{Cl}_{2}(2 \times 100 \mathrm{~mL})$, washed with a solution of $\mathrm{NaHCO}_{3}, \mathrm{NaCl}$, dried over anhydrous magnesium sulfate and the solvent evaporated. The residue was purified by column chromatography $(50 \%$ EtOAc in hexanes as eluent) to give the target compound.

2-(6-(2-Chloro-4-(trifluoromethyl)phenoxy)-1,2-dihydro-2,4-dioxoquinazolin-3(4H)-yl)- $\mathrm{N}$ (4-methylphenyl) acetamide (5d). Yield, 52\%, white solid, mp 280-281 ${ }^{\circ} \mathrm{C}$. ${ }^{1} \mathrm{H}$ NMR $\left(300 \mathrm{MHz}, \mathrm{CDCl}_{3}\right) \delta(\mathrm{ppm}) 2.27(\mathrm{~s}, 3 \mathrm{H}), 4.69(\mathrm{~s}, 2 \mathrm{H}), 7.14(\mathrm{~d}, J 8.7 \mathrm{~Hz}, 1 \mathrm{H}, \mathrm{Ar}-\mathrm{H}), 7.30-7.54(\mathrm{~m}$, 3H, Ar-H), 7.56-7.64 (m, 4H, Ar-H), 7.67 (d, J 8.7 Hz, 1H, Ar-H), 7.84 (s, 1H, Ar-H), 10.40 (s, 1H). Anal. Calcd. for $\mathrm{C}_{24} \mathrm{H}_{17} \mathrm{ClF}_{3} \mathrm{~N}_{3} \mathrm{O}_{4}$ : C, 57.21; H, 3.40; N, 8.34; Found: C, 57.11; H, 3.33; N, 8.31 .

Biological Testing. Three plant species: Barnyard (Echinochloa crusgalli), crabgrass (Digitaria sanguinalis), and velvetleaf (Abutilon theophrasti) were used for the test. The seeds were allowed to germinate and grow for 14 days. Test plants were selected for uniformity, size and stage of development and then treated with the test compound, returned to the greenhouse and 
watered. The plants not treated with the compound under evaluation were used as a comparison. The compound to be evaluated was dissolved in acetone and sprayed using a carrier volume equivalent to 187 liters per hectare at 1000 and $200 \mathrm{gha}^{-1}$. Two weeks after application of the test compounds, the state of the plants was observed. Each species was evaluated on a scale of 0-100 in which 0 equals no activity and 100 equals total control. The average control of the three plant species was calculated.

\section{Acknowledgements}

We gratefully acknowledge the National Key Project of Scientific and Technology of China for financial support.

\section{References}

1. Duke, S O; Lydon, J; Becerril, J M; Sherman, T D; Lehner, L P; Mastumoto, H. Weed Science 1991, 39, 465.

2. Duke, S O; Becerril, M J; Sherman, T D; Lydon, J; Matsumoto, H. Pestic. Sci. 1990, 30, 367.

3. Koch, M; Breithaupt, C; Kiefersauer, R; Freigang, J; Huber, R; Messerschmidt, A. The EMBO Journal 2004, 23, 1720.

4. Dyan, F E; Reddy, K N; Duke, S. O. In: Peroxidizing Herbicides; Boger, P., Wakabayashi, K. EDs; Springer, Berlin, 1999; pp141.

5. Duke, S. O.; Nandihalli, U. B.; Lee, H. J.; Duke, M. V. ACS Symp. Ser. 1994, 559, 191.

6. Wepplo, P. J.; Birk, J. H.; Lavanish, J. M.; Manfredi, M. C.; Nielsen, D. R. ACS Symp. Ser. 1995, 584, 136.

7. Ohki, A; Ohki, S; Koizumi, K; Kohno, H; Boger, P; Wakabayashi, K. J. Pestic. Sci. 1997, 22, 309 .

8. Sakagami, K.; Fukami, J.; Kawaguchi, N.; Niwada, S.;Sago, R.; Igai, K. JP. Pat. 05339239, 1993: Chem. Abstr. 1994, 120, 292147.

9. Sumida, M.; Niwata, S.; Fukami, H.; Tanaka, T.; Wakabayashi, K.; Boger, P. J. Agric. Food Chem. 1995, 43, 1929.

10. Lee, H. j.; Duke, M. V.; Brik, J. H.; Yamamoto, M.; Duke, S. O. J. Agric. Food Chem. 1995, 43, 2722.

11. Cordon, M. E.; Karp, G. M; Birk, Jeffrey, H. Eur. Pat. Appl. 459,133, 1991: Chem. Abstr. 1992, 117, 48332.

12. Barton, J. E.; Cartwright, D.; McCormack, D. Eur. Pat. Appl. 448,206, 1991: Chem. Abstr. 1992, 116, 21047. 
13. Chrystal, E. J.; Barton, J. E.; Cartwright, D.; Mathews, C. J. Eur. Pat. Appl. 442,655, 1991 : Chem. Abstr. 1991, 115, 207984.

14. Cartwright, D; Mathews, C. J. Brit. UK. Pat. Appl. 225,3847, 1992: Chem. Abstr. 1993, 118, 59578.

15. Karp, G. M.; Agric. R. D. J. Org. Chem. 1992, 57, 4765.

16. Dayan, F. E.; Allen, S. N. Pestic. Sci. 2000, 56, 717.

17. Condon, M.E.; Alvarado, S. I.; Arthen, F. J.; Birk, J. H.; Brady, T. E.; Crews, A.D; Marc, P. A.; Karp, G. M.; Lavanish, J. M.; Nielsen, D.R.; Lies, T. A. In: Synthesis and Chemistry of Agrochemicals IV. Baker, D. R.; Fenyes, J. G.; Basarab, G. S. Eds; ACS Symp. Ser. 1995, $584,122$.

18. Wepplo, P.; Birk, J. H.; Lavanish, J. M.; Manfredi, M. Nielsen, D. R. In: Synthesis and Chemistry of Agrochemicals IV. Baker, D. R; Fenyes, J. G.; Basarab, G. S. EDs; ACS Symp. Ser. 1995, 584, 149.

19. Naoko, K.; Jiichi, F; Shinjiro, N.; Ryuichi, S.; Fumio, F. JP. Pat. 06,316,568, 1994: Chem. Abstr. 1995, 122, 160660.

20. Fukami, J.; Hashimoto,M.; Sago, R.; Ikai, K. JP. Pat. 06,227,918, 1994: Chem. Abstr. 1995, $122,25875$.

21. Barton, J. E.; Cartwright, D.; Mathews, C. J. Brit. UK. Pat. Appl. 2,253,848, 1992: Chem. Abstr. 1993, 118, 124391.

22. Hunt, D. A.; Schwindeman, J. A. Eur. Pat. Appl. 299,446, 1989: Chem. Abstr. 1989, 110, 231433.

23. Theissen, R. J. Eur. Pat. Appl. 34,883, 1981: Chem. Abstr. 1982, 96, 122433.

24. Johnson, W. O. Eur. Pat. Appl. 20,052, 1980: Chem. Abstr. 1981, 94, 191921.

25. Li, F.; Feng, Y. Q.; Meng, Q. Q.; Li, W. H.; Li, Z. M.; Wang, Q. R. ARKIVOC 2006, (i), 40. 